\title{
The Apsidal Precession for Low Earth Sun Synchronized Orbits
}

\author{
Shkelzen Cakaj \\ Faculty of Electrical and Computing Engineering, \\ University of Prishtina, \\ Prishtina, Kosovo
}

\author{
Bexhet Kamo, Algenti Lala, Ilir Shinko, Elson Agastra \\ Faculty of Information Technology, \\ Polytechnic University of Tirana, \\ Tirana, Albania
}

\begin{abstract}
By nodal regression and apsidal precession, the Earth flattering at satellite low Earth orbits (LEO) is manifested. Nodal regression refers to the shift of the orbit's line of nodes over time as Earth revolves around the Sun. Nodal regression is orbit feature utilized for circular orbits to be Sun synchronized. A sun-synchronized orbit lies in a plane that maintains a fixed angle with respect to the Earth-Sun direction. In the low Earth Sun synchronized circular orbits are suited the satellites that accomplish their photo imagery missions. Nodal regression depends on orbital altitude and orbital inclination angle. For the respective orbital altitudes the inclination window for the Sun synchronization to be attained is determined. The apsidal precession represents major axis shift, respectively the argument of perigee deviation. The apsidal precession simulation, for inclination window of sun synchronized orbital altitudes, is provided through this paper.
\end{abstract}

\section{Keywords-LEO; satellite; orbit; apsidal precession}

\section{INTRODUCTION}

Orbital perturbation analyzes consider the satellite's orbit behavior under real space-ambient circumstances compared with ideal orbit mathematical model defined by Kepler parameters. Due to the gravitational forces of the Sun, other celestial bodies and also due to the Earth's flattering at both poles the perturbations are caused. Based on geopotential model the effect of the Earth flattering is determined as a sum of spherical harmonics, where the most dominated term is " $\mathrm{J} 2$ term" [1].

The Earth flattering is manifested by the nodal regression and apsidal precession. The apsidal precession is too early considered by Newton for celestial bodies, and lately the apsidal theorem is further more analytically considered, among them by authors under [2]. For satellites orbiting the Earth, the laws of celestial bodies' movement are applied. This concept is applied for the apsidal precession analysis of the Sun synchronized low Earth orbiting (LEO) satellites.

Under the second section, the nodal regression is considered and followed by terms the orbit Sun synchronization to be accomplished. Considering different initial low Earth orbit altitudes the inclination window for orbit Sun synchronization is determined. For the determined inclination window, the apsidal precession is simulated.

\section{NODAL REGRESSION}

The Earth rotates eastward. An orbit where the satellite moves in the same direction as the Earth's rotation is known as prograde or direct orbit. The inclination of a prograde orbit lies between $0^{\circ}$ and $90^{\circ}$. Most satellites are launched in a prograde orbit because the Earth's rotational velocity provides part of the orbital velocity with a consequent saving in launch energy. An orbit where the satellite moves in opposite direction to the Earth rotation is called retrograde orbit. The inclination of a retrograde orbit always lies between $90^{\circ}$ and $180^{\circ}$ [3], [4].

The Earth is not the spherical homogeneous body. Earth is characterized with a bulge at the equator, and a slight flattening at the both poles. The terrestrial potential at a point in space (in our case the point indicates a satellite) depends not only on the distance $r$ to the Earth's centre but also on the longitude and latitude of the point and the time. This happens due to irregularities of Earth's rotation and not homogenous Earth's mass distribution. This terrestrial potential depends much more on latitude than longitude, and it through geopotential coefficients $J_{n}$ is expressed. The $J_{2}$ term due to flattering of the Earth (about 20km) dominate all other terms. The values of these coefficients are given by different models. Based on GEM4 model it is [1]:

$$
J_{2}=1.0827 \cdot 10^{-3}
$$

To a first approximation, the orbital plane is fixed in space as the satellite orbits around the Earth. But, the potential generated by the non-spherical Earth causes variations of the orbital elements. Most effected orbital elements are the right ascension and the argument of perigee, as presented in Figure.1 [3], [4]. The right ascension of the ascending node $(\Omega)$ defines the location of the ascending and descending orbital crossing nodes (these two nodes make a line of nodes) with respect to a fixed direction in space. The fixed direction is Vernal Equinox. The Vernal Equinox is the direction of the line joining the Earth's center and the Sun on the first day of spring. The argument of perigee $(\omega)$ is the angle taken positively from $0^{\circ}$ to $360^{\circ}$ in the direction of the satellite's motion, between the direction of the ascending node and the perigee direction [3], [4].

Under the effect of terrestrial potential variation, the right ascension of the ascending node $\Omega$, shifts its position, so, the line of nodes which is in equatorial plane rotates about the center of the Earth, consequently shifting the orbital plane. Nodal regression refers to the shift of the orbital plane over time as Earth revolves around the Sun. Nodal regression is a very useful feature that is especially utilized for Low Earth circular orbits providing to them the Sun synchronization. 


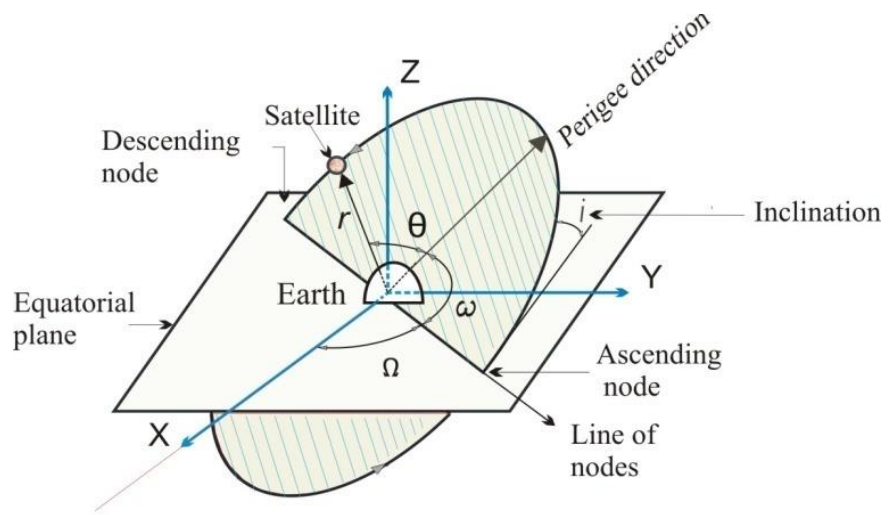

Vernal equinox

Fig. 1. Space orbital parameters

An approximate expression for the nodal rate regression of $\Omega$ due to time is expressed as [1]:

$$
\frac{d \Omega}{d t}=-\left(\frac{3}{2}\right) n_{0} A J_{2} \cos i
$$

Where:

$$
A=\frac{R_{E}^{2}}{a^{2}\left(1-e^{2}\right)^{2}}
$$

$R_{E}=6378 \mathrm{~km}$ is Earth's radius, $e$ is orbital eccentricity, $i$ is the inclination, $a$ is a semimajor axis of satellite's orbit and $n_{0}$ is mean movement of the satellite, as:

$$
n_{0}=\frac{2 \pi}{T}
$$

Where $T$ is the orbital period. For circular orbits it is $e=0$ and $a=r$, where $r$ is orbital radius of circular orbit. Orbital period for circular orbits is expressed as:

$$
T=2 \pi \sqrt{\frac{r^{3}}{\mu}}
$$

where $\mu=3.986005 \cdot 10^{5} \mathrm{~km}^{3} / \mathrm{s}^{2}$ is Earth's geocentric gravitational constant. For circular orbit yields:

$$
A=\frac{R_{E}^{2}}{r^{2}}
$$

Substituting Eqn. (4), Eqn. (5), and Eqn. (6) at Eqn. (2) and then considering values of $R_{E}, \mu$ and $J_{2}$, finally stems nodal regression expressed by inclination $i$ and orbital radius $r$. The nodal regression expressed in (\%day) is [5]:

$$
\Delta \Omega=-2.06474 \cdot 10^{14} \cdot \frac{\cos i}{r^{7 / 2}} \quad[\% / \mathrm{day}]
$$

The nodal regression for circular orbits depends upon orbit inclination and orbital altitude (radius). The nodal regression is zero in the case of the inclination angle being $90^{\circ}$.

When the orbit inclination angle is $i<90^{\circ}$ than deviation is negative, so according to Eqn. 2 the satellite orbital plane rotates in a direction opposite to the direction of the Earth's rotation. When the orbit inclination angle is $i>90^{\circ}$, then deviation is positive, so the satellite orbital plane rotates in the same direction as the direction of the Earth's rotation. From this stems that if the orbit is prograde the nodes slide westward, and if it is retrograde, the nodes slide eastward. This means that nodes (line of nodes) because of this effect move in opposite direction to the direction of satellite motion, hence the term nodal regression.

\section{ORBITAL SUN SYNCHRONIZATION}

LEO (Low Earth Orbiting) satellites have very wide applications, from astronomical purposes, remote sensing of oceans, Earth's climate changes or Earth's imagery with high resolution [6]. For photo imagery missions from satellites, in order the observed area to be treated under the same lighting (illumination) conditions, the observed area's position related to the Sun it is too important [7]. For these purposes the satellites are suited in LEO sun synchronized orbits [8].

A sun-synchronous orbit is one that lies in a plane that maintains a fixed angle with respect to the Earth-Sun direction. In other words, the orbital plane has a fixed orientation with respect to the Earth-Sun direction. Consequently, the angle between normal orbital plane vector and normal Sun vector is always kept constant throughout the year, as shown in Figure 2 [8].

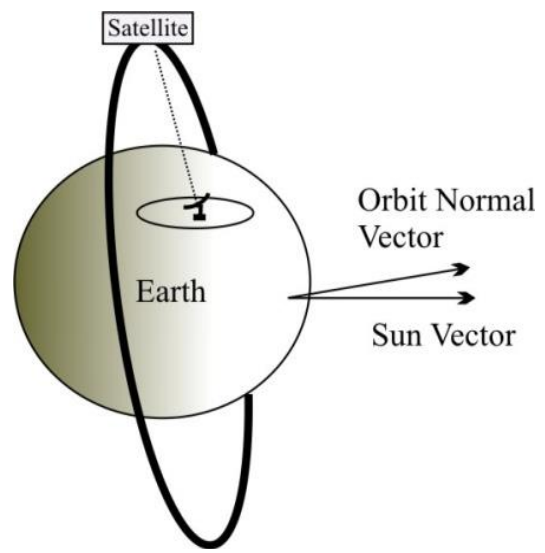

Fig. 2. Orbital Sun synchronization concept

As a result of this property, sun-synchronous orbits ensure that the satellite passes over a given location on Earth every time at the same local solar time, thereby guaranteeing almost the same illumination conditions, varying only with seasons. The satellite in sun-synchronized orbit ensures coverage of the whole surface of the Earth, since the appropriate orbit is quasipolar in nature [9]. The shift control method to keep the local time shift within an allowance range is given by [10].

The nodal regression effect is typical for LEO orbits. The further goal of this paper is to conclude about the inclination window of the nodal regression for different LEO orbit altitudes ensuring to be attained the orbital Sun synchronization. For simulation purposes are considered low Earth orbit altitudes from $600 \mathrm{~km}$ up to $1200 \mathrm{~km}$. LEOs have too low eccentricity which one can be considered $e \approx 0$ and then $a=r$. Thus, for altitudes from $600 \mathrm{~km}$ up to $1200 \mathrm{~km}$ and considering Earth's radius as $R_{E} \approx 6400 \mathrm{~km}$ yields out the 
orbits' radius range from $7000 \mathrm{~km}$ up to $7600 \mathrm{~km}$. Considering Eqn. 7, for this orbits' radius range it is calculated the nodal regression [\%day] for different inclination angles $i$. Results confirm that nodal regression for altitudes form $600 \mathrm{~km}$ up to $1200 \mathrm{~km}$ may range from $0^{\circ}$ up to $6.7^{\circ}$ as a function of inclination angle. Lower inclination causes higher deviation. For inclination of $90^{\circ}$ there is no nodal deviation [11].

The further step is inclination window determination for Sun synchronized feature. An orbital plane fixed with respect to the Earth effectively makes a $360^{\circ}$ rotation in space in a year (about 365.25 days) since the Earth itself rotates by $360^{\circ}$ around the Sun. This rate is equivalent to a rotation of the orbital plane of about 0.986 [\%day]. By choosing a pair of particular values of $i$ and $r$, it is possible to obtain an orbit for which the nodal regression varies each day by a quantity equal to the rotation of the Earth around the Sun [8]. Mathematically this is expressed as:

$$
\begin{gathered}
\frac{d \Omega}{d t}=0.9856^{\circ} / \text { day } \\
\Delta \Omega=-2.06474 \cdot 10^{14} \cdot \frac{\cos i}{r^{7 / 2}}=0.9856^{\circ} / \text { day }
\end{gathered}
$$

The angle between line of nodes of the orbits and the mean direction of the Sun obtained in this way remains constant throughout the year as presented in Figure 3.

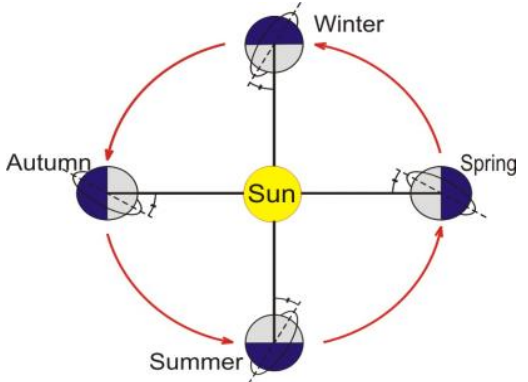

Fig. 3. Sun synchronization throughout seasons

So, the orbit normal vector and Sun vector keep the same angularity during the year. These orbits are known as Sun synchronized orbits.

Sun-synchronous orbits are a function of altitude, eccentricity and inclination. By solving the Eqn, 9 for orbital altitude of $600 \mathrm{~km}$ consequently for $a=r=7000 \mathrm{~km}$ under no eccentricity $(e=0)$ will get inclination for sun synchronization as:

$$
i_{1}=97.9^{\circ}
$$

and for orbital attitude of $1200 \mathrm{~km}$ consequently for $a=r=7600 \mathrm{~km}$ under no eccentricity $(e=0)$ will get inclination for sun synchronization as:

$$
i_{2}=100.5^{\mathrm{o}}
$$

Considering these values of inclination, and the range for lower and higher orbital attitudes, the nodal regression for the inclination range from $97^{\circ}$ up to $101^{\circ}$ it is presented in Table 1 and Figure 4 [11]. For the range of low Earth altitudes from
$600 \mathrm{~km}$ up to $1200 \mathrm{~km}$ the inclination window for the orbital Sun synchronization is:

$$
97.9^{\circ}<i<100.5^{\circ}
$$

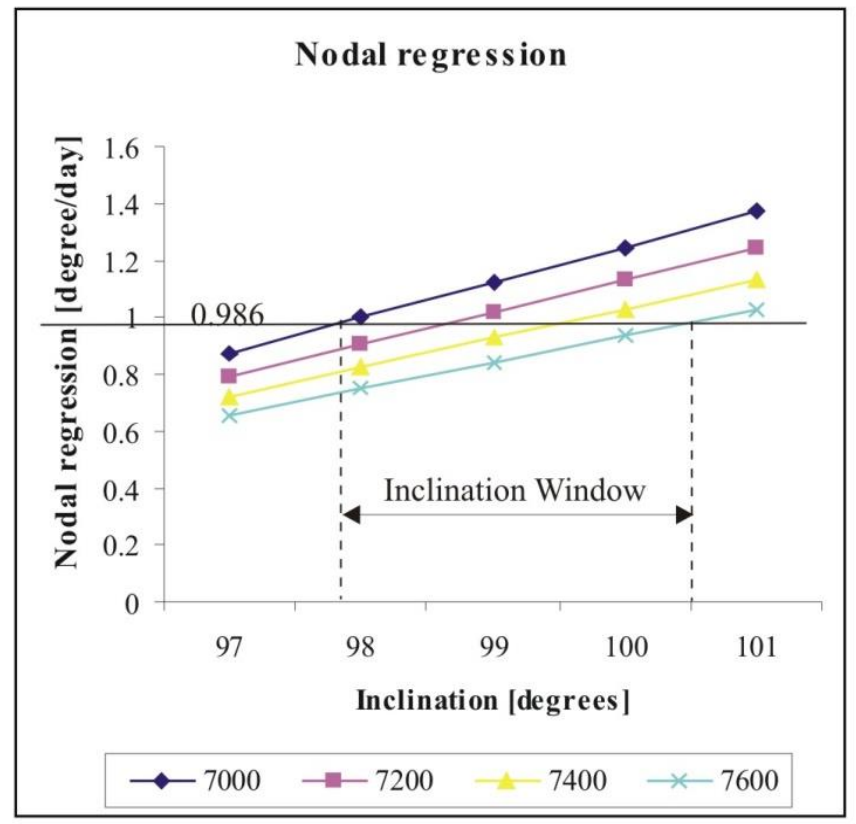

Fig. 4. Inclination window

\section{APSIDAL PRECESSION}

The position of the orbit major axis is defined by the argument of perigee. This parameter like the right ascension of the ascending node also undergoes natural perturbation due to equatorial bulge of Earth. The phenomenon is known as apsidal precession.

An approximate expression for the apsidal precession of perigee's argument $(\omega)$ over the time is expressed as [1]:

$$
\frac{d \omega}{d t}=\left(\frac{3}{4}\right) n_{0} A J_{2}\left(5 \cos ^{2} i-1\right)
$$

where all parameters on the right side of Eqn. (11) have the same meaning as for nodal regression expressed through Eqn. (1) up to Eqn. (6).

The aim of this paper is to conclude about the apsidal range which corresponds to inclination window for orbit Sun synchronization. This calculation leads toward the thrust vector to be applied in order to keep argument of perigee under in advance defined value, consequently keeping the health of the appropriate satellite link. Considering Eqn. (13), there is no apsidal precession if the orbital inclination fulfills the feature expressed as follows,

$$
5 \cos ^{2} i-1=0
$$

This happens under the inclination of $63^{\circ} 43^{\prime}$. This is exactly the inclination applied for Molnya elliptic orbit. The rotation of the perigee occurs in the direction opposite to the satellite motion if the inclination is greater than $63^{\circ} 43^{\prime}$ and in the same direction as the satellite motion if the inclination angle is less than $63^{\circ} 43^{\prime}$ [9]. 
Substituting Eqn. (4), Eqn. (5), and Eqn. (6) at Eqn. (11) and then considering values of $R_{E}, \mu$ and $J_{2}$, finally stems the apsidal precession expressed by inclination $i$ and orbital radius $r$. The apsidal precession expressed in (\%day) is:

$$
\Delta \omega=1.03237 \cdot 10^{14} \cdot \frac{\left(5 \cos ^{2} i-1\right)}{r^{7 / 2}}[\% \text { day }]
$$

The apsidal precession for circular orbits depends upon orbit inclination and orbit altitude (radius). The closer the satellite to Earth center, it is the larger apsidal precession. The apsidal precession is zero in the case of the inclination angle being $63^{\circ} 43^{\prime}$. Considering Eqn. (13), and orbits' radius range from $7000 \mathrm{~km}$ up to $7600 \mathrm{~km}$, it is calculated the apsidal precession [\%day] for inclination window which ensures low Earth Sun- synchronization. These results are given in Table I and Figure 5.

TABLE I. APSIDAL PRECESSION [ \% DAY ]

\begin{tabular}{|l|l|l|l|l|}
\hline \multirow{2}{*}{$\begin{array}{l}\text { Inclination } \\
{\left[{ }^{\mathrm{o}}\right]}\end{array}$} & \multicolumn{3}{|l|}{ Orbital radius [km] } \\
\cline { 2 - 5 } & 7000 & 7200 & 7400 & 7600 \\
\hline \hline 97 & 3.329 & 3.014 & 2.738 & 2.494 \\
\hline 98 & 3.248 & 2.943 & 2.673 & 2.435 \\
\hline 99 & 3.156 & 2.858 & 2.596 & 2.365 \\
\hline 100 & 3.054 & 2.766 & 2.513 & 2.289 \\
\hline 101 & 2.942 & 2.662 & 2.419 & 2.203 \\
\hline
\end{tabular}

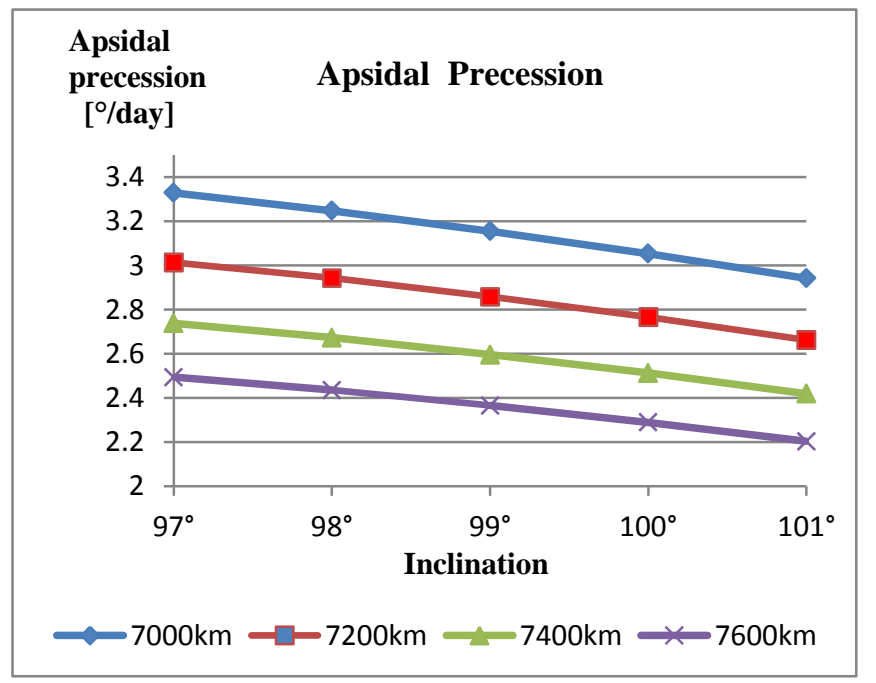

Fig. 5. Apsidal precession

\section{CONCLUSIONS}

Satellites dedicated for photo imagery missions are suited in low Earth Sun synchronized circular orbits. Nodal regression is the typical feature of low Earth circular orbits which enables orbital Sun synchronization. Sun synchronized orbits are retrograde orbits. Sun synchronization is achieved only for inclination within inclination window.

The apsidal precession for circular orbits depends upon orbit inclination and orbit altitude. The closer the satellite to the Earth center, it is the larger apsidal precession. For the Sun synchronization inclination window for altitudes from $600 \mathrm{~km}$ to $1200 \mathrm{~km}$, the low Earth circular orbits are faced with apsidal precession in the range of $2.2^{\circ}-3.3^{\circ}$.

\section{REFERENCES}

[1] G. Maral and M. Bousquet, "Satellite communication systems," John Willey \& Sons, Ltd, Chichester, England, 2002.

[2] S.R. Valluri, P.Yu, G..E. Smith and P.A. Wiegert, "An extension of Newtons apsodal precession theorem", Momthly Notices of Rolyal Astronomical Society (MNRAS), Vol. 358, No. 4, pp. 1273-1284, 2005.

[3] D. Roddy, "Satellite communications", McGraw Hill, New York, 2006.

[4] M. Richharia, (1999), "Satellite communication systems", McGraw Hill, New York, 1999.

[5] Basics of space flight: Orbital mechanics, pg. 14, www.braeunig.us/space/orbmech.htm, 12/4/2011

[6] J.E. Oberright, Satellite artificial, World Book Online Reference Center, World Book, Inc, 2004.

[7] S. Cakaj, W. Keim, K. Malaric, "Sun Noise Measurement at Low Earth Orbiting Satellite Ground Station", 47 $7^{\text {th }}$ International Symposium ELMAR 2005 focused on Multimedia Systems and Applications, IEEE, 8-10 June 2005, Zadar, Croatia, pp. 345 - 348.

[8] S. Cakaj, M. Fischer, A. L. Schotlz, "Sun synchronization of Low Earth Orbits (LEO) through inclination angle", $28^{\text {th }}$ IASTED International Conference on Modelling, Identification and Control, MIC 2009, Feb. 16-18, 2009, Innsbruck, Austria, pp. 155-161.

[9] A.K. Maini and V. Agrawal, "Satellite Technology-Principles and Applications", Wiley, UK, 2011.

[10] Y.Yong'an, et al., "Shift control method for the local time at descending node based on sun-synchronous orbit satellite", Journal of Systems Engineering and Electronics, Vol.20, No.1, pp.141-145, BIAI, 2009.

[11] S. Cakaj, B. Kamo, K. Malaric, "The Inclination Window for Low Earth Sun Synchronized Satellite Orbits", Transactions on Maritime Science Volume 2, Number 1, April 2013, Split, Croatia, pp. 15-19. 